Peter Quast

\title{
A pinching theorem for extrinsically symmetric submanifolds of Euclidean space
}

\author{
Received: 12 June 2004 / Published online: 15 October 2004
}

\begin{abstract}
We show that a compact connected manifold which can be immersed into $\mathbb{R}^{m}$ with almost parallel second fundamental form, admits an extrinsically symmetric immersion into $\mathbb{R}^{m}$.
\end{abstract}

\section{Introduction}

Extrinsically symmetric submanifolds of Euclidean space are immersed submanifolds which are invariant under the reflections at each of their normal spaces. The corresponding immersions will also be called extrinsically symmetric and are in fact covering maps. Extrinsically symmetric submanifolds of Euclidean space have been classified by Ferus showing that the only connected non-compact examples are products of compact connected extrinsically symmetric spaces and totally geodesic submanifolds (see [2]) and that the compact connected extrinsically symmetric spaces are exactly the symmetric orbits of $s$-representations (see [3], [4] and [1]). Not all simply connected compact symmetric spaces can be realized as extrinsically symmetric submanifolds of Euclidean space.

General symmetric spaces are locally characterized by the parallelity of the Riemannian curvature tensor. In [9] Strübing showed that compact connected extrinsically symmetric spaces in Euclidean space are even globally characterized by the local condition that the second fundamental form $\alpha$ is parallel w.r.t. the normal covariant derivative, i.e. $\nabla^{\perp} \alpha=0$. In this article we investigate the stability of the condition $\nabla^{\perp} \alpha=0$. Our main result, Theorem 2, states the following: If a compact connected Riemannian manifold is immersed into some Euclidean space with almost parallel second fundamental form, then it admits an extrinsically symmetric immersion. A similar theorem in the case of abstract symmetric spaces has been obtained by Katsuda [5], [6]. As in the papers of Katsuda, the proof of our main result (Theorem 2) is based on Gromov compactness and convergence and on an explicit bound showing how the parallelity of the second fundamental form measures extrinsic symmetry (Proposition 2).

P. Quast: Department of Mathematics, University of Fribourg, 1700 Fribourg, Switzerland Mathematics Subject Classification (2000): 53C20, 53C24, 53C30, 53C35, 53C40, 53C42 


\section{Preliminaries}

A real valued function $f$ defined on a bounded domain $\Omega$ of some Euclidean space is said to be of class $\mathcal{C}^{k, \alpha}, k \in \mathbb{N}, \alpha \in[0,1]$, if it is bounded in the $\mathcal{C}^{k, \alpha}$ Höldernorm:

$$
\|f\|_{k, \alpha}=\sum_{0 \leq|\beta| \leq k} \sup _{x \in \Omega}\left|\partial_{\beta} f(x)\right|+\sum_{|\beta|=k} \sup _{x \neq y} \frac{\left|\partial_{\beta} f(x)-\partial_{\beta} f(y)\right|}{|x-y|^{\alpha}} .
$$

A tensor on a compact manifold $M$ resp. a mapping between two manifolds is said to be of class $\mathcal{C}^{k, \alpha}$, if there are local coordinates such that in these coordinates its components are of class $\mathcal{C}^{k, \alpha}$. For a compact manifold $M$ we denote by $\mathcal{C}^{k, \alpha}\left(M, \mathbb{R}^{m}\right)$ the Hölder space of $\mathcal{C}^{k, \alpha}$ functions form $M$ to $\mathbb{R}^{m}$. We have the following embedding theorem for Hölder spaces:

Proposition 1. Let $M$ be a compact manifold, $k_{1}, k_{2}$ be two positive integers and $0 \leq \alpha_{1}, \alpha_{2} \leq 1$ such that

$$
k_{1}+\alpha_{1}>k_{2}+\alpha_{2} \text {. }
$$

Then the canonical embedding

$$
\mathcal{C}^{k_{1}, \alpha_{1}}\left(M, \mathbb{R}^{m}\right) \longrightarrow \mathcal{C}^{k_{2}, \alpha_{2}}\left(M, \mathbb{R}^{m}\right)
$$

is compact, i.e. any bounded sequence in $\mathcal{C}^{k_{1}, \alpha_{1}}\left(M, \mathbb{R}^{m}\right)$ has a convergent subsequence in $\mathcal{C}^{k_{2}, \alpha_{2}}\left(M, \mathbb{R}^{m}\right)$.

By $\mathcal{M}(\Lambda, d, v, n)$ we denote the class of $n$-dimensional compact Riemannian manifolds $M$ with bounded sectional curvature $|K| \leq \Lambda^{2}$, diameter $\operatorname{diam}(M) \leq d$ and lower bound on the volume $\operatorname{vol}(M) \geq v$. M. Gromov, S. Peters and R. Greene and $\mathrm{H}$. Wu (see [8, Appendix]) provided the following convergence result for sequences of Riemannian manifolds in $\mathcal{M}(\Lambda, d, v, n)$ :

Theorem 1. Let $\left(M_{i}, g_{i}\right)_{i \in \mathbb{N}}$ be a sequence in $\mathcal{M}(\Lambda, d, v, n)$ and let $\left.\alpha^{\prime} \in\right] 0,1[$ be fixed. Then there exists a subsequence $\left(M_{i_{j}}, g_{i_{j}}\right)_{j \in \mathbb{N}}$ and a smooth manifold $M$ equipped with a Riemannian metric $g$ of class $\mathcal{C}^{1, \alpha^{\prime}}$ such that the following holds: There is an integer $j_{0}$ such that for all $j \geq j_{0}$ there are $\mathcal{C}^{\infty}$-diffeomorphisms $f_{i_{j}}: M \longrightarrow M_{i_{j}}$ such that the sequence of pullback metrics $\left(f_{i_{j}}^{*} g_{i_{j}}\right)_{j \in \mathbb{N}}$ on $M$ converge to $g$ in the $\mathcal{C}^{1, \alpha}$ topology $\left(0<\alpha<\alpha^{\prime}\right)$.

Let $\|\cdot\|$ be norms such that $\|A x\| \leq\|A\| \cdot\|x\|$ where $A$ is a matrix and $x$ a vector, e.g. the canonical Euclidean norm on $\mathbb{R}^{n}$ and the Frobenius norm $\|A\|=$ $\left\|\left(a_{i j}\right)\right\|=\sqrt{\sum_{i, j=1}^{n} a_{i j}^{2}}$ on the set of $n \times n$-matrices. For further use, we state the following Gronwall inequality:

Lemma 1. Let $f, b:[0, \tau] \longrightarrow \mathbb{R}^{n}$ and $A=\left(a_{i, j}\right):[0, \tau] \longrightarrow \mathbb{R}^{n \times n}$ be smooth functions. Assume that the functions $A(t)$ and $b(t)$ are bounded on $[0, \tau]$, i.e. $\|A(t)\| \leq A_{0}$ and $\|b(t)\| \leq b_{0}$. If $f^{\prime}(t)=A(t) f(t)+b(t)$, then

$$
\|f(t)\| \leq\|f(0)\| e^{A_{0} t}+\frac{b_{0}}{A_{0}}\left(e^{A_{0} t}-1\right) .
$$




\section{Estimates}

In this section let $\left(M^{n}, g\right)$ be a compact connected $n$-dimensional Riemannian manifold and

$$
f:(M, g) \longrightarrow\left(\mathbb{R}^{m}, g_{\text {can }}\right)
$$

an isometric immersion into the $m$-dimensional Euclidean space with its canonical metric. The pullback bundle $f^{*} T \mathbb{R}^{m}$ over $M$ splits as a direct sum of the tangent bundle $T M$ and the normal bundle $v M$, i.e. $f^{*} T \mathbb{R}^{m}=T M \oplus v M$, both equipped with a bundle metric. The metric on $v M$ is denoted by $g^{\perp}$. Let $\bar{\nabla}$ denote the canonical derivative in $\mathbb{R}^{m}$ and $\nabla$ the Levi-Civita connection on $M$. The normal bundle $\nu M$ is equipped with a metric connection $\nabla^{\perp}$ defined as the normal component $\nabla_{X}^{\perp} \xi=\left(\bar{\nabla}_{X} \xi\right)^{\perp}$. Here and in the following, vectors tangent to $M$ will be denoted by capital Roman letters and normal vectors by Greek ones. The second fundamental form $\alpha$ of $f$ is defined by $\alpha(X, Y)=\bar{\nabla}_{X} Y-\nabla_{X} Y$ and the corresponding shape operator $A$ by $A_{\xi} X=\nabla_{X}^{\perp} \xi-\bar{\nabla}_{X} \xi$. The second fundamental form and the shape operator are related by $g\left(A_{\xi} X, Y\right)=g^{\perp}(\alpha(X, Y), \xi)$. Moreover, $R$ denotes the Riemannian curvature tensor, $K$ the sectional curvature and $\operatorname{inj}(M)$ the injectivity radius of $M$.

\subsection{The Gauss equation}

Recall the Gauss equation in Euclidean space:

$$
R(X, Y) Z=A_{\alpha(Y, Z)} X-A_{\alpha(X, Z)} Y .
$$

Using the relation between the second fundamental form and the shape operator, this equation can be rewritten as

$$
\langle R(X, Y) Z, W\rangle=\langle\alpha(Y, Z), \alpha(X, W)\rangle-\langle\alpha(X, Z), \alpha(Y, W)\rangle,
$$

where $\langle\cdot, \cdot\rangle$ denotes the canonical scalar product in Euclidean space. For a tensor $T$ we denote by $\|T\|_{0}$ the supremum of the norm of this tensor with unit vectors as arguments.

Lemma 2. 1. $\|\alpha\|_{0}=\|A\|_{0}$;

2. $\|K\|_{0} \leq 2 \cdot\|\alpha\|_{0}^{2}$;

3. $\left\|\nabla^{\perp} \alpha\right\|_{0}=\|\nabla A\|_{0}$;

4. $\|\nabla R\|_{0} \leq 4 \cdot\|\alpha\|_{0} \cdot\left\|\nabla^{\perp} \alpha\right\|_{0}$.

Proof. We denote the unit tangent bundle over $M$ by $S M$ and the unit normal bundle by $v_{1} M$. To obtain the first equation we notice that

$$
\begin{aligned}
\|A\|_{0}^{2} & =\sup \left\{\left\|\left\langle A_{\xi} X, A_{\xi} X\right\rangle\right\| ; \quad X \in S M, \xi \in v_{1} M\right\} \\
& =\sup \left\{\left\|\left\langle\alpha\left(X, A_{\xi} X\right), \xi\right\rangle\right\| ; X \in S M, \xi \in v_{1} M\right\} \\
& \leq\|A\|_{0} \cdot \sup \left\{\left\|\left\langle\alpha\left(X, A_{\xi} X \cdot\left\|A_{\xi} X\right\|^{-1}\right), \xi\right\rangle\right\| ; X \in S M, \xi \in v_{1} M\right\} \\
& \leq\|A\|_{0} \cdot\|\alpha\|_{0} .
\end{aligned}
$$


Thus $\|A\|_{0} \leq\|\alpha\|_{0}$. The opposite inequality can be proved in the same manner.

Using the Gauss equation we get

$$
\begin{aligned}
\|K\|_{0}= & \sup \{|K(X, Y)| ; X, Y \in S M\} \\
= & \sup \{|\langle R(X, Y) Y, X\rangle| ; X, Y \in S M, X \perp Y\} \\
= & \sup \left\{\left|\langle\alpha(X, X), \alpha(Y, Y)\rangle-\|\alpha(X, Y)\|^{2}\right| ; X, Y \in S M, X \perp Y\right\} \\
\leq & \sup \left\{\|\alpha(X, X)\| \cdot\|\alpha(Y, Y)\|+\|\alpha(X, Y)\|^{2} ;\right. \\
& X, Y \in S M,, X \perp Y\} \\
\leq & 2\|\alpha\|_{0}^{2} .
\end{aligned}
$$

This proves the second inequality.

To obtain the third equation we proceed analogously to the first one using the fact that $\left\langle\left(\nabla_{X}^{\perp} \alpha\right)(Y, Z), \xi\right\rangle=\left\langle\left(\nabla_{X} A\right)_{\xi} Y, Z\right\rangle$ :

$$
\begin{aligned}
\|\nabla A\|_{0}^{2}= & \sup \left\{\left\|\left(\nabla_{X} A\right)_{\xi} Y\right\|^{2} ; X, Y \in S M, \xi \in v_{1} M\right\} \\
= & \sup \left\{\left|\left\langle\left(\nabla_{X}^{\perp} \alpha\right)\left(Y,\left(\nabla_{X} A\right)_{\xi} Y\right), \xi\right\rangle\right| ; X, Y \in S M, \xi \in v_{1} M\right\} \\
\leq & \sup \left\{\|\left(\nabla_{X}^{\perp} \alpha\right)\left(Y,\left(\nabla_{X} A\right)_{\xi} Y\|\cdot\| \xi \| ; X, Y \in S M, \xi \in v_{1} M\right\}\right. \\
= & \sup \left\{\left\|\left(\nabla_{X} A\right)_{\xi}\right\| \cdot\left\|\left(\nabla_{X}^{\perp} \alpha\right)\left(Y,\left(\nabla_{X} A\right)_{\xi} Y \cdot\left\|\left(\nabla_{X} A\right)_{\xi} Y\right\|^{-1}\right)\right\| ;\right. \\
& \left.X, Y \in S M, \xi \in v_{1} M\right\} \\
\leq & \|\nabla A\|_{0} \cdot\left\|\nabla^{\perp} \alpha\right\|_{0} .
\end{aligned}
$$

Therefore $\|\nabla A\|_{0} \leq\left\|\nabla^{\perp} \alpha\right\|_{0}$. The opposite inequality can be obtained similarly.

For the proof of the fourth inequality, we may assume that $V, X, Y, Z$ are radially $\nabla$-parallel unit vector fields around a point $p$ in $M$. Then at the point $p$ we have $\left(\nabla_{V} R\right)(X, Y, Z)=\nabla_{V}(R(X, Y) Z),\left(\nabla_{X} A\right)_{\xi} Y=\nabla_{X}\left(A_{\xi} Y\right)-A_{\nabla_{X}^{\perp} \xi} Y$ and $\left(\nabla_{X}^{\perp} \alpha\right)(Y, Z)=\nabla_{X}^{\perp}(\alpha(Y, Z))$. Therefore,

$$
\begin{aligned}
\|\nabla R\|_{0}= & \sup \left\{\left\|\nabla_{V}(R(X, Y) Z)\right\|\right\} \\
= & \sup \left\{\left\|\nabla_{V}\left(A_{\alpha(Y, Z)} X\right)-\nabla_{V}\left(A_{\alpha(X, Z)} Y\right)\right\|\right\} \\
\leq & \sup \left\{\left\|\left(\nabla_{V} A\right)_{\alpha(Y, Z)} X\right\|+\left\|A_{\nabla_{V}^{\perp} \alpha(Y, Z)} X\right\|+\left\|\left(\nabla_{V} A\right)_{\alpha(X, Z)} Y\right\|\right. \\
& \left.+\left\|A_{\nabla_{V}^{\perp} \alpha(X, Z)} Y\right\|\right\} \\
= & 2 \cdot\|\alpha\|_{0} \cdot\|\nabla A\|_{0}+2 \cdot\|A\|_{0} \cdot\left\|\nabla^{\perp} \alpha\right\|_{0} \\
= & 4 \cdot\|\alpha\|_{0} \cdot\left\|\nabla^{\perp} \alpha\right\|_{0},
\end{aligned}
$$

by the first and the third equation.

\subsection{Lower bounds on the injectivity radius and the volume}

Lemma 3. Assume that $\|\alpha\| \leq \Lambda$ for some $\Lambda>0$. Then the injectivity radius and the volume of $M$ satisfy:

$$
\operatorname{inj}(M) \geq \frac{\pi}{\Lambda \sqrt{2}} \quad \text { and } \operatorname{vol}(M) \geq\left(\frac{1}{\Lambda \sqrt{2}}\right)^{n} \cdot \operatorname{vol}\left(S^{n}, g_{0}\right),
$$

where $\left(S^{n}, g_{0}\right)$ is the $n$-dimensional unit sphere in Euclidean space. 
Proof. Let $\gamma:[0, L] \longrightarrow M$ be a shortest simply closed non-constant geodesic parameterized by arclength, i.e. $\gamma$ is a shortest geodesic such that $\gamma(0)=$ $\gamma(L), \dot{\gamma}(0)=\dot{\gamma}(L)$ and $\gamma(t) \neq \gamma(s)$ for $s, t \in] 0, L[$. Then the curvature $\kappa$ of the curve $f \circ \gamma$ in $\mathbb{R}^{m}$ is given by

$$
\kappa=\left\|\bar{\nabla}_{(f \circ \gamma)^{\prime}}(f \circ \gamma)^{\prime}\right\|=\left\|\alpha\left(f_{*}\left(\gamma^{\prime}\right), f_{*}\left(\gamma^{\prime}\right)\right)\right\|
$$

and therefore $\kappa \leq \Lambda$. By Fenchel's theorem on the total curvature of a curve in Euclidean space we get

$$
2 \pi \leq \int_{0}^{L} \kappa(t) \mathrm{d} t \leq \Lambda \cdot L
$$

By Lemma 2 the sectional curvature satisfies $\|K\| \leq 2 \Lambda^{2}$. Therefore the infimum $t_{0}$ of all first conjugate values of points in $M$ is greater or equal to $\frac{\pi}{\Lambda \sqrt{2}}$ and we get a lower bound on the injectivity radius using a result of Klingenberg (see [8, p. 111]):

$$
\operatorname{inj}(M)=\min \left(t_{0}, \frac{L}{2}\right) \geq \min \left(\frac{\pi}{\Lambda \sqrt{2}}, \frac{\pi}{\Lambda}\right)=\frac{\pi}{\Lambda \sqrt{2}} .
$$

The lower bound on the volume follows by Berger's isoembolic inequality (see [8, p. 252]).

\subsection{Second fundamental form and extrinsic symmetry}

The normal covariant derivative of the second fundamental form measures how far an immersion $f$ is away from being extrinsically symmetric: Let $p$ be a point on $M$ and let $r_{p} \in \operatorname{Iso}\left(\mathbb{R}^{m}\right)$ be the reflection along the normal space at $f(M)$ in $f(p)$. Let $\gamma$ be a geodesic in $M$ parameterized by arclength emanating from $p$ and let $\tilde{\gamma}(t)=\gamma(-t)$.

Proposition 2. Assume that $\left\|\nabla^{\perp} \alpha\right\|_{0} \leq \varepsilon$, then we have:

$$
\left\|\left(r_{p} \circ f \circ \gamma-f \circ \tilde{\gamma}\right)(t)\right\| \leq \varepsilon \cdot k_{\varepsilon, T, \Lambda, m, n}(t) \text { for } 0 \leq t \leq T,
$$

where $k_{\varepsilon, T, \Lambda, m, n}(t)=k_{1}\left(\frac{1}{k_{2}} e^{k_{2} t}-t-\frac{1}{k_{2}}\right)$ with the constants $k_{1}=\frac{2 T \sqrt{m}}{\varepsilon T+\Lambda}$ and $k_{2}=c_{m, n}(\varepsilon T+\Lambda)$ depending on $\varepsilon, T, m, n$ and the upper bound $\Lambda$ of $\|\alpha\|_{0}$.

Proof. This proof is based on a method due to Olmos and Sánchez [7]. The basic idea goes back to Strübing [9].

Recall that $\operatorname{Dr}_{p}(p)(X)=-X$ if $X$ is tangent to $f(M)$ and $\operatorname{Dr}_{p}(p)(\xi)=\xi$ if $\xi$ is normal to $f(M)$. Now we construct a frame along $f \circ \gamma$ in the following way: Choose an orthonormal basis $\left\{e_{1}, \ldots, e_{m}\right\}$ of $T_{f(p)} \mathbb{R}^{m}$ such that

$e_{1}=(f \circ \gamma)^{\prime}(0), \quad\left\{e_{1}, \ldots, e_{n}\right\} \subset T_{f(p)} f(M), \quad\left\{e_{n+1}, \ldots, e_{m}\right\} \subset v_{f(p)} f(M)$.

Then we define the frame field $\left\{E_{1}(t), \ldots, E_{m}(t)\right\}$ along $f \circ \gamma$ in the following way: $E_{1}(t), \ldots, E_{n}(t)$ are the $\nabla$-parallel vector fields along $f \circ \gamma$ defined by 
$e_{1}, \ldots, e_{n}$ and $E_{n+1}(t), \ldots, E_{m}(t)$ are the $\nabla^{\perp}$-parallel vector fields along $f \circ \gamma$ given by $e_{n+1}, \ldots, e_{m}$. Notice that $E_{1}(t)=(f \circ \gamma)^{\prime}(t)$. Using the functions

$$
h_{i j}^{E}: \mathbb{R} \longrightarrow \mathbb{R} ; \quad t \longmapsto\left\langle E_{i}^{\prime}(t), E_{j}(t)\right\rangle,
$$

where $E_{i}^{\prime}(t)=\left(\bar{\nabla}_{(f \circ \gamma)^{\prime}} E_{i}\right)(t)$, we get the following system of differential equations:

(E)

$$
\left\{\begin{array}{l}
(f \circ \gamma)^{\prime}(t)=E_{1}(t) ; \\
E_{i}^{\prime}(t)=\sum_{j=1}^{m} h_{i j}^{E}(t) E_{j}(t), \quad i=1, \ldots, m \\
\text { with coefficients } \\
h_{i j}^{E}(t)=\left\{\begin{array}{l}
0 ; \quad i, j \in\{1, \ldots, n\} \text { or } i, j \in\{n+1, \ldots, m\} \\
\left\langle\alpha\left(E_{1}(t), E_{i}(t)\right), E_{j}(t)\right\rangle ; \quad i \in\{1, \ldots, n\}, j \in\{n+1, \ldots, m\} \\
-\left\langle\alpha\left(E_{1}(t), E_{j}(t)\right), E_{i}(t)\right\rangle ; i \in\{n+1, \ldots, m\}, j \in\{1, \ldots, n\}
\end{array}\right. \\
\text { and initial conditions } \\
(f \circ \gamma)(0)=p ; \quad E_{i}(0)=e_{i}, \quad i=1, \ldots, m .
\end{array}\right.
$$

Along $r_{p} \circ f \circ \gamma$ we consider the frame field $\left\{F_{1}, \ldots, F_{m}\right\}$ defined by $F_{i}(t)=$ $r_{p_{*}}\left(E_{i}(t)\right)$. Since $r_{p}$ is an isometry of $\mathbb{R}^{m}$, we get the following system of differential equations:

$(F)\left\{\begin{array}{l}\left(r_{p} \circ f \circ \gamma\right)^{\prime}(t)=F_{1}(t) ; \\ F_{i}^{\prime}(t)=\sum_{j=1}^{m} h_{i j}^{E}(t) F_{j}(t), \quad i=1, \ldots, m \\ \text { with the same coefficients as in }(E) \text { and with initial conditions } \\ \left(r_{p} \circ f \circ \gamma\right)(0)=p ; F_{i}(0)=f_{i}=\left(r_{p}\right)_{*}\left(e_{i}\right)=\left\{\begin{array}{ll}-e_{i}, & i=1, \ldots, n \\ e_{i}, & i=n+1, \ldots, m\end{array} .\right.\end{array}\right.$

Analogously we construct a frame field $\left\{G_{1}(t), \ldots, G_{m}(t)\right\}$ along $f \circ \tilde{\gamma}$ : $G_{1}(t), \ldots, G_{n}(t)$ are the $\nabla$-parallel vector fields along $f \circ \tilde{\gamma}$ defined by $f_{1}, \ldots, f_{n}$ and $G_{n+1}(t), \ldots, G_{m}(t)$ are the $\nabla^{\perp}$-parallel vector fields along $f \circ \tilde{\gamma}$ defined by $f_{n+1}, \ldots, f_{m}$. Notice that $G_{1}(t)=(f \circ \tilde{\gamma})^{\prime}(t)$. As before we define functions

$$
h_{i j}^{G}: \mathbb{R} \longrightarrow \mathbb{R} ; \quad t \longmapsto\left\langle G_{i}^{\prime}(t), G_{j}(t)\right\rangle
$$

where $G_{i}^{\prime}(t)=\left(\bar{\nabla}_{(f \circ \tilde{\gamma})^{\prime}} G_{i}\right)(t)$ and we get the following system of differential equations:

$(G)$

$$
\left\{\begin{array}{l}
(f \circ \tilde{\gamma})^{\prime}(t)=G_{1}(t) ; \\
G_{i}^{\prime}(t)=\sum_{j=1}^{m} h_{i j}^{G}(t) G_{j}(t), \quad i=1, \ldots, m \\
\text { with coefficients } \\
h_{i j}^{G}(t)=\left\{\begin{array}{l}
0 ; \quad i, j \in\{1, \ldots, n\} \text { or } i, j \in\{n+1, \ldots, m\} \\
\left\langle\alpha\left(G_{1}(t), G_{i}(t)\right), G_{j}(t)\right\rangle ; \quad i \in\{1, \ldots, n\}, j \in\{n+1, \ldots, m\} \\
-\left\langle\alpha\left(G_{1}(t), G_{j}(t)\right), G_{i}(t)\right\rangle ; i \in\{n+1, \ldots, m\}, j \in\{1, \ldots, n\}
\end{array}\right. \\
\text { and initial conditions } \\
(f \circ \tilde{\gamma})(0)=p ; \quad G_{i}(0)=f_{i}, \quad i=1, \ldots, m .
\end{array}\right.
$$


Now consider the difference between the coefficients

$$
d_{i j}(t)=h_{i j}^{E}(t)-h_{i j}^{G}(t)
$$

To get an estimate on $\left|d_{i j}^{\prime}\right|$ it is sufficient to assume that $i=1, \ldots, n$ and $j=n+1, \ldots, m$. In this case we have

$$
d_{i j}(t)=\left\langle\alpha\left(E_{1}(t), E_{i}(t)\right), E_{j}(t)\right\rangle-\left\langle\alpha\left(G_{1}(t), G_{i}(t)\right), G_{j}(t)\right\rangle .
$$

In particular $d_{i j}(0)=0$ and

$$
d_{i j}^{\prime}=\left\langle\left(\nabla_{(f \circ \gamma)^{\prime}}^{\perp} \alpha\right)\left(E_{1}, E_{i}\right), E_{j}\right\rangle-\left\langle\left(\nabla_{(f \circ \tilde{\gamma})^{\prime}}^{\perp} \alpha\right)\left(G_{1}, G_{i}\right), G_{j}\right\rangle .
$$

Now suppose $0 \leq t \leq T$, then we get the estimates $\left|d_{i j}^{\prime}\right| \leq 2\left\|\nabla^{\perp} \alpha\right\|_{0} \leq 2 \cdot \varepsilon$ and therefore

$$
\left|d_{i j}(t)\right|=\left|\int_{0}^{t} d_{i j}^{\prime}(s) \mathrm{d} s+d_{i j}(0)\right| \leq \int_{0}^{t}\left|d_{i j}^{\prime}(s)\right| \mathrm{d} s \leq \int_{0}^{T} 2 \cdot \varepsilon \mathrm{d} s \leq 2 \varepsilon T .
$$

The difference of the systems (F) and (G) gives rise to a new system of differential equations:

$$
\left(D_{1}\right)\left\{\begin{array}{l}
\left(r_{p} \circ f \circ \gamma-f \circ \tilde{\gamma}\right)^{\prime}(t)=F_{1}(t)-G_{1}(t) ; \\
F_{i}^{\prime}(t)-G_{i}^{\prime}(t)=\sum_{j=1}^{m} h_{i j}^{E}(t)\left(F_{j}(t)-G_{j}(t)\right)+\sum_{j=1}^{m} d_{i j}(t) G_{j}(t), \\
\text { with initial conditions } \\
\left(r_{p} \circ f \circ \gamma-f \circ \tilde{\gamma}\right)(0)=0 ; \quad F_{i}(0)-G_{i}(0)=0, \quad i=1, \ldots, m .
\end{array}\right.
$$

Rewriting a subsystem, we get the ordinary differential equation:

$$
\left(D_{2}\right)\left\{\begin{array}{l}
F^{\prime}(t)-G^{\prime}(t)=H^{E}(t)(F(t)-G(t))+D(t) G(t), \\
\text { with initial conditions } \\
F(0)-G(0)=0, \quad i=1, \ldots, m
\end{array}\right.
$$

where $F=\left(F_{1}, \ldots, F_{m}\right)^{T} \in \mathbb{R}^{m^{2}}, G=\left(G_{1}, \ldots, G_{m}\right)^{T} \in \mathbb{R}^{m^{2}}$,

$$
H^{E}(t)=\left(\begin{array}{lll}
h_{11}^{E} I_{m} & \ldots & h_{1 m}^{E} I_{m} \\
\vdots & \ddots & \vdots \\
h_{m 1}^{E} I_{m} & \ldots & h_{m m}^{E} I_{m}
\end{array}\right) \quad \text { and } \quad D(t)=\left(\begin{array}{lll}
d_{11} I_{m} & \ldots & d_{1 m} I_{m} \\
\vdots & \ddots & \vdots \\
d_{m 1} I_{m} & \ldots & d_{m m} I_{m}
\end{array}\right) \text {. }
$$

The following inequality holds:

$$
\left|h_{i j}^{E}(t)\right| \leq \varepsilon \cdot T+\Lambda .
$$

In fact, consider the case $i=\{1, \ldots, n\}$ and $j \in\{n+1, \ldots, m\}$. As

$$
\left(h_{i j}^{E}\right)^{\prime}=\bar{\nabla}_{(f \circ \gamma)^{\prime}}\left\langle\alpha\left(E_{1}, E_{i}\right), E_{j}\right\rangle=\left\langle\left(\nabla_{(f \circ \gamma)^{\prime}}^{\perp} \alpha\right)\left(E_{1}, E_{i}\right), E_{j}\right\rangle,
$$

we have

$$
\left|\left(h_{i j}^{E}\right)^{\prime}\right| \leq\left\|\nabla^{\perp} \alpha\right\| \cdot\left\|E_{j}\right\| \leq \varepsilon,
$$


and therefore

$$
\left|h_{i j}^{E}(t)\right|=\left|\int_{0}^{t}\left(h_{i j}^{E}\right)^{\prime}(s) \mathrm{d} s+h_{i j}^{E}(0)\right| \leq \int_{0}^{T} \varepsilon \mathrm{d} s+\left|h_{i j}^{E}(0)\right| \leq \varepsilon \cdot T+\Lambda,
$$

since $\left|h_{i j}^{E}(0)\right|=\left|\left\langle\alpha\left(e_{1}, e_{i}\right), e_{j}\right\rangle\right| \leq\|\alpha\|_{0} \leq \Lambda$.

Since the matrices $H^{E}$ and $D$ have at most $2 n m(m-n)$ non-vanishing components, we get, using the above estimates for $\left|d_{i j}\right|$ and $\left|h_{i j}^{E}(t)\right|$ and the fact that the vectors $G_{i}$ have length one, the following estimates of the Frobenius norms:

$$
\left\|H^{E}(t)\right\| \leq(\varepsilon T+\Lambda) \sqrt{2 n m(m-n)}=c_{m, n}(\varepsilon T+\Lambda)=: k_{2}
$$

with $c_{m, n}=\sqrt{2 n m(m-n)}$ and

$$
\|D(t) G(t)\| \leq\|D(t)\| \cdot\|G(t)\| \leq 2 \varepsilon \operatorname{Tm} \sqrt{2 n(m-n)}=: \kappa .
$$

Since $\|F(0)-G(0)\|=0$, the Gronwall inequality of Lemma 1 implies

$$
\|F(t)-G(t)\| \leq \varepsilon \cdot k_{1}\left(e^{k_{2} t}-1\right)
$$

where $k_{1}=\frac{\kappa}{\varepsilon k_{2}}=\frac{2 T \sqrt{m}}{\varepsilon T+\Lambda}$. From the system $\left(D_{2}\right)$ we get by the fundamental theorem of calculus:

$$
\begin{aligned}
\left\|\left(r_{p} \circ f \circ \gamma-f \circ \tilde{\gamma}\right)(t)\right\| & =\left\|\int_{0}^{t}\left(F_{1}(s)-G_{1}(s)\right) \mathrm{d} s\right\| \\
& \leq \int_{0}^{t}\left\|F_{1}(s)-G_{1}(s)\right\| \mathrm{d} s \\
& \left.\leq \int_{0}^{t} \| F(s)-G(s)\right) \| \mathrm{d} s \\
& \leq \int_{0}^{t} \varepsilon \cdot k_{1}\left(e^{k_{2} s}-1\right) \mathrm{d} s \\
& =\varepsilon \cdot k_{1}\left(\frac{1}{k_{2}} e^{k_{2} t}-t-\frac{1}{k_{2}}\right) .
\end{aligned}
$$

Remark. The bound $\varepsilon \cdot k_{\varepsilon, T, \Lambda, m, n}(t)$ in the above proposition converges to zero if $\varepsilon$ tends to zero. In particular, $\nabla^{\perp} \alpha=0$ implies extrinsic symmetry, i.e. the inner geodesic reflection and outer normal reflection coincide. Notice that, since $M$ is compact, it cannot be immersed into any Euclidean space as totally geodesic submanifold. Therefore the second fundamental form cannot be identically zero, i.e. $\Lambda>0$.

\section{The result}

Let $\mathcal{M}_{\mathrm{im}}(\Lambda, d, n, m)$ be the set of all pairs $(M, f)$ consisting of a compact connected $n$-dimensional smooth manifold $M^{n}$ and an immersion $f$ of $M$ into the $m$-dimensional Euclidean space $\left(\mathbb{R}^{m}, g_{\text {can }}\right)$ with the following property: Measured in the pullback metric $f^{*} g_{\text {can }}$, the diameter of $M$ is bounded form above by $d$ and the second fundamental from of $f$ satisfies $\|\alpha\|_{0} \leq \Lambda$. 
Recalling the definition of $\mathcal{M}(\Lambda, d, v, n)$ and using Lemma 2 and Lemma 3, we notice that, if $(M, f)$ is an element of $\mathcal{M}_{\text {im }}(\Lambda, d, n, m)$, then $\left(M, f^{*} g_{\text {can }}\right)$ lies in $\mathcal{M}\left(\Lambda \sqrt{2}, d,(\Lambda \sqrt{2})^{-n} \operatorname{vol}\left(S^{n}, g_{0}\right), n\right)$.

Theorem 2. There exists a constant $\varepsilon>0$ depending only on $\Lambda, n$ and $m$ with the following property: If a pair $(M, f)$ in $\mathcal{M}_{\mathrm{im}}(\Lambda, d, n, m)$ satisfies

$$
\left\|\nabla^{\perp} \alpha\right\|_{0} \cdot d^{3} \leq \varepsilon
$$

then there exists an extrinsically symmetric immersion of $M$ into the $m$-dimensional Euclidean space $\left(\mathbb{R}^{m}, g_{\text {can }}\right)$.

Proof. Multiplying $f$ by a constant, we can assume that $d=1$. As we have to consider subsequences several times, we do not introduce a special notation in order to keep the proof readable.

Assume by contradiction that for each positive integer $i$, there exists a pair $\left(M_{i}, f_{i}\right)$ in $\mathcal{M}_{\mathrm{im}}(\Lambda, 1, n, m)$ such that the second fundamental for $\alpha_{i}$ of $f_{i}$ satisfies $\left\|\nabla_{i}^{\perp} \alpha_{i}\right\|_{0} \leq \frac{1}{i}$, but $M_{i}$ does not admit an extrinsically symmetric immersion into $\left(\mathbb{R}^{m}, g_{\text {can }}\right)$. Here $\nabla_{i}$ denotes the Levi-Civita connection and $\nabla_{i}^{\perp}$ the normal connection of the pullback metric. Since $\left(M_{i}, f_{i}^{*} g_{\text {can }}\right)$ is a sequence in $\mathcal{M}\left(\Lambda \sqrt{2}, 1,(\Lambda \sqrt{2})^{-n} \operatorname{vol}\left(S^{n}, g_{0}\right), n\right)$, we can assume by Theorem 1 , that after passing to a subsequence there are a smooth manifold $M$ equipped with a $\mathcal{C}^{1, \alpha^{\prime}}$ Riemannian metric $g$ and diffeomorphisms $h_{i}: M \rightarrow M_{i}$ such that the metrics $g_{i}:=h_{i}^{*}\left(f_{i}^{*} g_{\text {can }}\right)$ converge to $g$ in the $\mathcal{C}^{1, \alpha}$-topology, $0<\alpha<\alpha^{\prime}<1$. Therefore the diameter of $(M, g)$ is also bounded by 1 . Let $\|\cdot\|_{0}^{g_{i}}$ denote the supremum norm w.r.t. the metric $g_{i}$. Then by Lemma 2 we get $\left\|\nabla_{i} R_{i}\right\|_{0}^{g_{i}} \leq 4 \cdot \Lambda \cdot \frac{1}{i}$. The results of Katsuda (see [5] and [6]) now imply that the Riemannian manifold $(M, g)$ is locally symmetric. In particular $g$ is a smooth metric on $M$. We denote by $\nabla$ the Levi-Civita connection corresponding to $g$.

Setting $\tilde{f}_{i}:=f_{i} \circ h_{i}$, we get a new sequence $\left(M, \tilde{f}_{i}\right)$ in $\mathcal{M}_{\text {im }}(\Lambda, 1, n, m)$. Now we fix a point $p_{0}$ on $M$. By composition with an appropriate translation of $\mathbb{R}^{m}$, we can assume that $\tilde{f}_{i}\left(p_{0}\right)=0$. The $\mathcal{C}^{2}$-norm of $\tilde{f}_{i}$ on $\left(M, g_{i}\right)$ is defined by $\left\|\tilde{f}_{i}\right\|_{\mathcal{C}^{2}\left(\left(M, g_{i}\right), \mathbb{R}^{m}\right)}=\left\|\tilde{f}_{i}\right\|_{0}^{g_{i}}+\left\|\mathrm{d} \tilde{f}_{i}\right\|_{0}^{g_{i}}+\left\|\nabla_{i} \mathrm{~d} \tilde{f}_{i}\right\|_{0}^{g_{i}}$, where the second covariant derivative of $\tilde{f}_{i}$ should be interpreted componentwise. Notice that this norm is equivalent to the norm of Proposition 1. Since $M$ is connected and $\tilde{f}_{i}\left(p_{0}\right)=0$, the maximum of $\tilde{f}_{i}$ is not greater than the diameter $d=1$ of $M$. And because $\tilde{f}_{i}$ is an isometric immersion, the supremum norm of its derivative equals 1 . The second fundamental form coincides with the Hessian of the immersion, so that $\left\|\nabla_{i} \mathrm{~d} \tilde{f}_{i}\right\|_{0}=$ $\left\|\alpha_{i}\right\|_{0}$. Summing up we get $\left\|\tilde{f}_{i}\right\|_{\mathcal{C}^{2}\left(\left(M, g_{i}\right), \mathbb{R}^{m}\right)} \leq 2+\Lambda$. Since the sequence $\left(g_{i}\right)$ converges to $g$ in the $\mathcal{C}^{1, \alpha}$-topology and $\nabla_{i} \mathrm{~d} h$ involves only the first derivative of $g_{i}$, the sequence $\left(\nabla_{i} \mathrm{~d} h\right)$ converges to $\nabla \mathrm{d} h$ for any function $h$ and the norms $\|\cdot\| \|_{0}^{g_{i}}$ converge pointwise to $\|\cdot\|_{0}$, the supremum norm corresponding to the metric $g$. Therefore the sequence $\left(\tilde{f}_{i}\right)$ is bounded in $\mathcal{C}^{2}\left((M, g), \mathbb{R}^{m}\right)$ as well. By the embedding theorem for Hölder spaces there exist a function $f \in \mathcal{C}^{1, \beta}\left((M, g), \mathbb{R}^{m}\right), 0<\beta<1$ and a subsequence of $\left(\tilde{f}_{i}\right)$ converging to a function $f$ in $\mathcal{C}^{1, \beta}\left((M, g), \mathbb{R}^{m}\right)$. From now on we restrict our attention to this subsequence $\left(M, \tilde{f}_{i}\right)$. Since the metric $g$ is smooth, its normal coordinates on $M$ are compatible with the smooth structure of 
$M$, and, since $\tilde{f}_{i}$ converges to $f$ in the $\mathcal{C}^{1}$-topology, $f$ is an isometric immersion of $(M, g)$ into $\mathbb{R}^{m}$. Considering normal coordinates on $(M, g)$, we see that $f$ is the identity in these charts and therefore smooth.

To get a contradiction, it remains to be shown that $f$ is extrinsically symmetric. Let $X$ be a unit vector of $T_{p} M, p \in M$ w.r.t. the metric $g$ and $X_{i}$ the unit vector w.r.t. $g_{i}$ obtained by rescaling $X$. Using the notations of Proposition 2, let $\gamma_{i}$ be the geodesic on $\left(M, g_{i}\right)$ defined by $X_{i}, \gamma$ the geodesic on $(M, g)$ defined by $X, r_{p_{i}}$ the reflection at the normal space at $\tilde{f}_{i}(M)$ in $\tilde{f}_{i}(p)$ and $r_{p}$ the reflection at the normal space at $\tilde{f}(M)$ in $\tilde{f}(p)$. By the regularity properties of the convergence of the metrics and the immersions, the distance between $\gamma_{i}$ and $\gamma$ as well as the distance between $\tilde{\gamma}_{i}$ and $\tilde{\gamma}$ tend to zero, as the differential equation for geodesics involves only the first derivative of the metric. Moreover, since the reflections $r_{p_{i}}$ involve only the first derivative (more precisely the position of the tangent space) and the value of the respective immersions at $p$, they converge towards $r_{p}$. Thus we have by Proposition 2

$$
\left\|\left(r_{p} \circ f \circ \gamma-f \circ \tilde{\gamma}\right)(t)\right\|=\lim _{i \rightarrow \infty}\left\|\left(r_{p_{i}} \circ \tilde{f}_{i} \circ \gamma_{i}-\tilde{f}_{i} \circ \tilde{\gamma}_{i}\right)(t)\right\|=0 .
$$

But this means that the immersion $f$ is extrinsically symmetric.

Acknowledgements. I am most grateful to J.-H. Eschenburg, P. Ghanaat and E. A. Ruh for valuable discussions and helpful remarks. This work was supported by the Swiss National Science Foundation Grants 20-67619.02 and PBFR-106367.

\section{References}

[1] Eschenburg, J.-H., Heintze, E.: Extrinsic symmetric spaces and orbits of s-representations. Manuscr. Math. 88, 517-524 (1995)

[2] Ferus, D.: Produkt-Zerlegung von Immersionen mit paralleler zweiter Fundamentalform. Math. Ann. 211, 1-5 (1974)

[3] Ferus, D.: Immersions with parallel second fundamental form. Math. Z. 140, 87-93 (1974)

[4] Ferus, D.: Symmetric submanifolds of Euclidean space. Math. Ann. 247, 81-93 (1980)

[5] Katsuda, A.: A pinching problem for locally homogeneous spaces. Lect. Notes in Math. 1201, 150-155 (1986)

[6] Katsuda, A.: A pinching problem for locally homogeneous spaces. J. Math. Soc. Japan 14 (1), 57-74 (1989)

[7] Olmos, C., Sánchez, C.: A geometric characterization of the orbits of $s$-representations. J. Reine Angew. Math. 420, 195-202 (1991)

[8] Sakai, T.: Riemannian Geometry. Providence, American Mathematical Society 1996

[9] Strübing, M.: Symmetric submanifolds of Riemannian manifolds. Math. Ann. 245, 37-44 (1979) 